\title{
The influence of caregivers' knowledge and understanding of asthma aetiology on domiciliary management of children with asthma
}

\author{
Wern Fern Soo ${ }^{1}$, MMed, FCFP, Ngiap Chuan $\underline{\operatorname{Tan}}^{1,2}$, MMed, FCFP
}

INTRODUCTION Asthma is a common childhood disease, and paediatric patients with asthma rely on caregivers to administer domiciliary asthma care. This study aimed to explore the knowledge, understanding, perceptions and main concerns of caregivers and its influence on their home management of children with asthma.

METHODS Data from 14 caregivers of children with asthma was collected during three focus group discussions held in two polyclinics in Singapore. The collected data was analysed using standard content analysis and classified into themes.

RESULTS The caregivers' main concerns included the perceived effects of infection, food and exercise on children with asthma. Several caregivers considered the disease to be infectious and had a lower threshold for physician consultation, as they believed that any delay in treating the infection would be detrimental to the child's health. Some also perceived asthma to be episodic and self-limiting, and that their children could 'outgrow' it. Many caregivers believed that asthma could be modified by abstinence from, or intake of, certain foods. Others had the perception that sports, including swimming, would worsen asthma. These perceptions resulted in unnecessary restrictions of the children's diet and activities. Most caregivers were unaware of influenza vaccination as a preventive measure to reduce triggers due to respiratory viral infections.

CONCLUSION We found that the caregivers' perceptions of asthma aetiology, its triggers and preventive measures affected their help-seeking behaviour and care of children with asthma. Healthcare professionals managing paediatric patients with asthma should recognise such caregiver misperceptions, and take a proactive approach to rectify and bridge the gaps in caregivers' knowledge and understanding of the disease.

Keywords: asthma, caregiver, knowledge, understanding

\section{INTRODUCTION}

Asthma is a common childhood disease. Caregivers (e.g. parents) are the key link between healthcare providers and children with asthma. Paediatric patients with asthma rely on caregivers to administer asthma care at home. Caregivers are also relied on to provide information on the child's condition to the healthcare provider. Many caregivers have varying domiciliary practices in their management of children with asthma. This is related to their understanding, knowledge and perceptions of asthma, which are often influenced by their fears and concerns, their prior experiences and the cultural health beliefs of their community. In other words, caregivers' understanding, knowledge and perceptions of asthma are likely to affect their management of children with asthma. DeWalt et al had shown that among children attending outpatient respiratory clinics in North Carolina Children's Hospital, low parental health literacy was linked to increased emergency department visits, hospitalisations and missed school. ${ }^{(1)}$

In many oriental societies, the development of cough and phlegm in the presence of asthma is believed to be due to an imbalance of 'yin' and 'yang', leading to a deficiency of 'qi' (air) - a concept originating from Taoism and other Asian cultures. External forces, such as a cold environment, a lack of rest and foods labelled as 'cold' (e.g. oranges), are perceived to be able to upset the 'qi' balance, aggravating asthma. As such, many Asians believe that perfect homeostasis can be achieved by balancing 'yin' ('coolness') and 'yang' ('heat') using traditional medication. The consumption of either Cordyceps or crocodile meat is seen as a way to boost 'yang qi', thus ameliorating asthma symptoms. ${ }^{(2)}$ However, evidence of its effectiveness is lacking. More information is required to understand such practices among caregivers of children with asthma in Singapore's multiethnic Asian community.

A recent study in the United States reported that children with asthma took part in fewer sports than their peers. ${ }^{(3)}$ Lang et al found that one fifth of parents of children with asthma felt that sports was dangerous for their children, with a quarter of these parents afraid that their child would become sick after exercising. ${ }^{(4)}$ Lai et al showed that parents expressed their concerns that swimming and other forms of exercise could aggravate asthma. ${ }^{(5)}$ Locally, little is known about the views of caregivers on the impact of exercise on children with asthma. While the avoidance of triggers and other preventive measures are recommended in most clinical practice guidelines on

${ }^{1}$ SingHealth Polyclinics - Bukit Merah, ${ }^{2}$ Duke-NUS Graduate Medical School, Singapore

Correspondence: Dr Soo Wern Fern, Associate Consultant, Bukit Merah Polyclinic, Block 163, \#04-3565, Bukit Merah Central, Singapore 150163. soo.wern.fern@singhealth.com.sg 
asthma, ${ }^{(6)}$ there is a paucity of information pertaining to the measures taken by local caregivers to prevent exposure to triggers in children with asthma. Furthermore, evidence-based preventive measures may not correlate to caregivers' perceptions. This may lead to suboptimal management of children with asthma and place an unnecessary economic burden on society. ${ }^{(7)}$

The present study thus aimed to explore the knowledge, understanding, perceptions and main concerns of caregivers of children with asthma in Singapore, and how these affect the home management of children with asthma. The results of this study will provide information that allows healthcare professionals, such as family physicians and asthma nurse counsellors, to rectify any misinformation and misperceptions on the part of the caregiver during asthma education programmes.

\section{METHODS}

This study was part of a larger study with a sequential mixed methodology design, comprising two segments - a qualitative segment, followed by a questionnaire survey conducted between 2008 and 2010. The present study presents the findings of the first segment. Focus group discussions (FGDs), which were moderated by us, were used to explore issues that were hitherto not well understood, as FGSs were a suitable and appropriate method.

Caregivers of children who had asthma and were aged 4-15 years old were identified on a case-encounter basis at two polyclinics (in Bukit Merah and Pasir Ris). These caregivers were invited to participate in the FGDs. Caregivers were defined as parents or guardians of children with asthma, who looked after the children for at least six hours daily. Caregivers were selected to participate in the study based on their ability to communicate in either English or any of the major local languages (i.e. Mandarin, Malay or Tamil). The participants were briefed on the objectives of the study and written informed consent was obtained. Ideas reached saturation after three FGDs.

FGDs were conducted in English and Mandarin, which were the main languages spoken by the participants. Audio recordings of the FGDs were transcribed into text. Responses given in Mandarin were translated into English. The collected qualitative data was subsequently analysed using NVivo 9 (QSR International, Doncaster, VIC, Australia). Themes that emerged after standard content analysis were deliberated and reconciled. Issues related to caregivers' perceptions and understanding of asthma and its management are presented in this study. The second segment of the larger study, which the present study was a part of, was a questionnaire survey of the caregivers' management of asthma in children. The questionnaire was based on the data gathered from the qualitative segment (i.e. the present study). The results of the second segment were reported in a separate publication. The study was approved by the SingHealth Polyclinics Institutional Review Board (IRB\# SHP-2008/02/001).
Table I. Demographic profile of caregivers who participated in the three focus group discussions (FGDs).

\begin{tabular}{lcclll}
\hline Participant & FGD & Age (yrs) & Gender & Role & Ethnicity \\
\hline 1 & 1 & 36 & Female & Mother & Malay \\
2 & 1 & 40 & Female & Mother & Chinese \\
3 & 1 & 36 & Female & Mother & Chinese \\
4 & 1 & 31 & Female & Mother & Malay \\
5 & 1 & 49 & Female & Mother & Malay \\
6 & 2 & 41 & Female & Mother & Indian \\
7 & 2 & 43 & Female & Mother & Chinese \\
8 & 2 & 43 & Female & Mother & Indian \\
9 & 2 & 44 & Female & Mother & Chinese \\
10 & 3 & 40 & Female & Mother & Chinese \\
11 & 3 & 36 & Male & Father & Chinese \\
12 & 3 & 34 & Female & Mother & Chinese \\
13 & 3 & 41 & Female & Mother & Malay \\
14 & 3 & 38 & Female & Mother & Chinese \\
\hline
\end{tabular}

\section{RESULTS}

Three FGDs were held separately in November 2008, and January and March 2009, with a total of 14 participants. Table I shows the demographic characteristics of these caregivers. Table II illustrates the main perceptions of the caregivers in this study, with documentation of some of their comments.

We found that caregivers perceived asthma to be caused by chest infections, which could affect a child's lungs if left untreated. Some caregivers also felt that, with time, children would outgrow their asthma. This is largely due to their own misconception or prior interaction with other family members with asthma. Asthma was also perceived as a disease that was not genetically linked and could be overcome by 'willpower', which would thus be self-limiting. It was noted that most caregivers in the study would avoid giving their children cold drinks, citrus foods, chocolates, sweets, nuts and milk, as these foods were perceived as triggers of asthma attacks. These misperceptions were sometimes reinforced by healthcare providers. While some caregivers were not entirely convinced about the health benefits of certain types of food with regard to asthma, caregivers were willing to try out health supplements and traditional medicine treatment. Common herbs and supplements cited by caregivers as being able to help improve asthma included Cordyceps, white gingko, crocodile meat, cod liver oil, goat milk products and Malay herbs. We also found that most of the caregivers in our study would not allow their child to participate in strenuous sports. Most of the caregivers were also not familiar with preventive measures that could be taken prior to exercise, and had conflicting views about children with asthma swimming. When one participant (Participant 5) was asked to confirm whether her child's asthma improved after swimming for the past one year, she replied in the affirmative. The caregivers were aware that dust exacerbates asthma and is a common trigger of asthma attacks, and some had taken steps to reduce their child's exposure to dust. Most 
Table II. Caregivers' perceptions and comments.

\begin{tabular}{|c|c|c|c|}
\hline Perception & Participant & FGD & Comment \\
\hline \multirow[t]{2}{*}{$\begin{array}{l}\text { Asthma is viewed as } \\
\text { an infectious, episodic } \\
\text { disease that can be } \\
\text { outgrown. }\end{array}$} & 4 & 1 & $\begin{array}{l}\text { "[children with asthma] have flu or cough for a few days, become infected, and asthma } \\
\text { will develop [...] the lungs become smaller, which makes it hard for them to breathe." } \\
\text { "I took care of my brother [...] the experience with him showed that asthma does go } \\
\text { away [...] now he's an athlete. He's running every day. No problem [is triggered] with } \\
\text { soft drinks or running, or whatever." }\end{array}$ \\
\hline & 8 & 2 & "If your child's asthma is well controlled, he will outgrow asthma." \\
\hline \multirow[t]{2}{*}{$\begin{array}{l}\text { Food is believed to have } \\
\text { a significant impact on } \\
\text { asthma symptoms }\end{array}$} & 5 & 1 & $\begin{array}{l}\text { "I saw this doctor who told me to avoid citrus fruits like oranges, [and drinks like] } \\
\text { 'Ribena'. [The doctor said to not give these types of food] to him because [they] will } \\
\text { cause a lot of phlegm. [...] I didn't give my son oranges." }\end{array}$ \\
\hline & 11 & 3 & $\begin{array}{l}\text { "[Based on] recommendations in magazines or books, such foods do no harm. For } \\
\text { example, crocodile meat, to me, is just another form of meat. I would try and see if it } \\
\text { works, rather than omit this kind of food." }\end{array}$ \\
\hline \multirow{3}{*}{$\begin{array}{l}\text { Exercises are } \\
\text { largely perceived to } \\
\text { aggravate asthma }\end{array}$} & 5 & 1 & $\begin{array}{l}\text { "For my child, I wouldn't allow very 'hard' (strenuous) exercise, like football. Only } \\
\text { swimming [is alright].” }\end{array}$ \\
\hline & 8 & 2 & $\begin{array}{l}\text { "[...] about swimming. If your kids have asthma, you refrain from soaking [them] too } \\
\text { long in the water. I don't know whether it is a myth or fact that swimming helps [...] } \\
\text { too long in the water, it is not good for asthmatic kids. For the chest, it will affect the } \\
\text { lungs, and on the other hand, it is good because it opens up the lungs when he moves." }\end{array}$ \\
\hline & 12 & 3 & $\begin{array}{l}\text { "Not very sure. l'd been to one clinic: the doctor told me that by swimming you can } \\
\text { control and minimise the asthma (exacerbation). Actually the answer is no, he said." }\end{array}$ \\
\hline $\begin{array}{l}\text { Generally aware of } \\
\text { triggers but less familiar } \\
\text { with some of the } \\
\text { evidence-based preventive } \\
\text { measures to reduce } \\
\text { asthma exacerbation. }\end{array}$ & 9 & 2 & $\begin{array}{l}\text { "Certain things like you know, your bedsheet, your house, have to be free of dust." } \\
\text { "Sometimes the children are very allergic, you know. Don't use carpet at home [...], } \\
\text { no soft toys, all these things. Certain things I can carry out." }\end{array}$ \\
\hline
\end{tabular}

caregivers were unaware of influenza vaccination as a prophylactic measure to reduce influenza-related complications in children with asthma. When asked whether they felt influenza vaccination could be an effective preventive measure in children with asthma, Participant 1 said that she did not think so. However, these caregivers were found to be willing to consider such preventive measures if they were provided with evidence to support its benefits.

In summary, the main points derived from the study are as follows: (a) asthma is often viewed as an infectious, episodic disease that can be outgrown; (b) food is generally believed to have a significant impact on asthma symptoms; (c) exercise is largely perceived to aggravate asthma; and (d) caregivers are generally aware of triggers, but are less familiar with some evidence-based preventive measures that can reduce asthma exacerbation.

\section{DISCUSSION}

The results of the present study demonstrated caregivers' personal perceptions of asthma aetiology. We observed that perceived triggers (e.g. infection, type of food consumed, and exercise) influenced the domiciliary care of children with asthma. The caregivers in the present study were also found to be less familiar with some evidence-based preventive measures to reduce asthma exacerbation. Some of them perceived asthma as a contagious disease. In a different survey of caregivers of children with asthma, up to $37 \%$ of caregivers were of the opinion that asthma could be spread from one person to another. ${ }^{(8)}$ This perceived communicable nature of asthma could engender stigma, placing considerable social and psychological strain on these children, which may lead to the development of low self-esteem.

As caregivers perceive the 'infectious' nature of asthma to be risky and detrimental to the health of the child, they have a lower threshold for bringing their child to seek treatment from a doctor. This help-seeking behaviour is made easier in the local community, as access to care is readily available in Singapore. Similar findings were also noted in a qualitative study of paediatric asthma caregivers in Hong Kong. ${ }^{(9)}$ Such misperceptions adversely affect caregivers' therapeutic options, which may lead to inappropriate treatment with antibiotics and other anti-infective medications. Although viral upper respiratory tract infection is a common trigger of asthma exacerbation, healthcare providers should make the effort to educate caregivers and correct the misconception that an asthma attack is in itself contagious. However, as both asthma and upper 
respiratory tract infection present with similar symptoms such as cough, caregivers may find it difficult to differentiate the two conditions.

A possible way to reduce asthma triggers is to vaccinate against influenza. However, the present study revealed that most caregivers were unaware of such a preventive measure. The reluctance and sense of scepticism of those who were aware of such a prophylactic measure could be due to the limited effectiveness of this vaccination against all respiratory viral infections, as it targets only the prevailing strains of influenza in the community. Nevertheless, caregivers indicated their willingness to vaccinate their children against influenza if they are provided with evidence of its benefits. Communication of the benefits of vaccination can be effected via individual or group asthma education programmes designed to rectify caregivers' misunderstanding and misperceptions of asthma triggers and influenza vaccination. Heightened media attention will also help to disseminate such information. While influenza vaccination is recommended in most asthma clinical practice guidelines, further prospective research should be carried out to determine the impact of vaccination on the various health outcomes of children with asthma.

We found that most of the caregivers who participated in the present study did not regard asthma as a chronic disease. They expected children with asthma to 'outgrow' the condition. Thus, they did not perceive a need for continuous monitoring and treatment of the condition. In a survey conducted on caregivers with children attending asthma clinics in the United Kingdom, South Asian parents were found to be more likely to believe that their children's asthma would 'get better'. ${ }^{(10)}$ Wong et al reported that $54 \%$ of caregivers of children with asthma believed that asthma could be cured; these parents did not realise the chronicity of the condition, resulting in premature termination of long-term preventive therapy with inhaled controller medication. ${ }^{(9)}$ These misconceptions should be corrected with appropriate asthma counselling.

Many caregivers in the present study perceived food to be major asthma trigger. They were found to have placed unfounded restrictions of a variety of food on children with asthma. A number of caregivers also believed in, and had resorted to, traditional remedies or complementary therapies in an attempt to improve the child's asthma status. This finding is consistent with the results of two Hong Kong studies. ${ }^{(5,9)}$ In a British qualitative study by Shaw et al, complementary therapies were perceived by caregivers of children with asthma as an integral part of the 'holistic' approach in disease treatment due to prior experiences of benefits from such therapies for asthma or other health problems. ${ }^{(11)}$ Such complementary therapies were viewed as 'natural' with less side effects than Western medicine, leading to wider acceptance by caregivers and patients with asthma. However, there is a need for healthcare providers to present balanced information to caregivers, pointing out that certain foods may be an asthma trigger among children with asthma and known food allergy, ${ }^{(12)}$ and that there is currently insufficient evidence to support the perception that common food substances such as citrus foods, cold drinks, chocolates and sweets act as asthma triggers that impact asthma exacerbation. Further large-scale trials need to be conducted to determine the validity of the aforementioned perceptions and to determine whether traditional remedies and alternative medicine are effective in preventing asthma exacerbations. The execution of such studies, however, will be challenging due to ethical considerations.

In the present study, most caregivers were found to restrict children with asthma from strenuous exercise. Breathlessness secondary to poor fitness of the child with asthma is often interpreted as exercise-induced asthma by caregivers, leading to inactivity being imposed on the child. In contrast to caregivers' misconceptions, studies have shown that swimming is beneficial for children with asthma. Wicher et al showed that for both children and adolescents with asthma who underwent a threemonth swimming program, their bronchial hyperresponsiveness, as measured using the methacholine test, was significantly reduced compared to that in non-swimmers, which remained unchanged. ${ }^{(13)}$ Although trichloramines, a by-product of chlorine commonly found in high quantities in indoor pools, when inhaled or in contact with the skin of swimmers may induce asthma attacks, this is rarely a problem when swimming in outdoor pools due to the better ventilation. ${ }^{(14)}$ Exercise is important for the normal growth and development of a child. With appropriate education on the use of inhaled bronchodilator medication before exercise to reduce exerciseinduced bronchoconstriction, exercise can remain an integral part of normal life for children with asthma. Swimmers with asthma and swimming-induced dyspnoea should be encouraged to swim in well-maintained, hygienic pools to reduce the effects of chemical trigger. Nevertheless, caregivers should recognise that children with asthma should not overexert themselves during asthma exacerbations or periods of acute illness.

Patient education is a key component of asthma management. In addition to patient education, educating caregivers improves their understanding of the disease, leading to better management of children with asthma. The findings of the present study would be useful in planning education strategies for caregivers of children with asthma. The success of asthma education programmes hinges on the assessment of the caregivers' level of knowledge and the correction of any misperceptions that can interfere with appropriate asthma management. Healthcare professionals also need to proactively address caregivers' fears and unwarranted concerns. In view of the limited healthcare and time resources in busy clinical practices, innovative use of multimedia platforms and help from other allied health professionals such as nurses and pharmacists should be considered for the delivery of asthma education. The present study revealed that a few caregivers were confused as a result 
of inconsistent advice from different healthcare professionals. Healthcare professionals should keep themselves updated so that they are able to deliver appropriate advice based on current medical evidence.

A limitation of the present study is that the prevalence of the issues raised by the caregivers was not determined. As the present study was a qualitative study, it also did not allow for the issues raised by the caregivers in the FGDs to be verified against their actual practices at home. The recruitment of participants for the FGDs solely from the polyclinics, being one of several primary healthcare providers, could be a source of selection bias. However, we were constrained by the approved protocol from the institution's review board.

In conclusion, understanding caregivers' perceptions of asthma aetiology, triggers and preventive measures plays a pivotal role in the effort to achieve optimal delivery of care to children with asthma. Other measures contributing to the improved care of children with asthma include specially tailored asthma education programmes that focus on bridging caregivers' gaps in knowledge and rectifying erroneous beliefs with regard to asthma and its management.

\section{ACKNOWLEDGEMENTS}

The authors are grateful to SingHealth Polyclinics and Ngee Ann Polytechnic for providing seed-funding for the study, and to Agnes Ngoh and Marina Tan for their assistance in executing the focus group discussions.

\section{REFERENCES}

1. DeWalt DA, Dilling MH, Rosenthal MS, Pignone MP. Low parental literacy is associated with worse asthma care measures in children. Ambul Pediatr 2007; 7:25-31.
2. Liu W. Traditional Chinese Medicine Information Page. Am Acad Acupuncture Oriental Medicine. In: Traditional Chinese Medicine/TCM and Asthma [online]. Available at: www.tcmpage.com/hpasthma.html. Accessed April 16, 2013.

3. Glazebrook C, McPherson AC, Macdonald IA, et al. Asthma as a barrier to children's physical activity: implications for body mass index and mental health. Pediatrics 2006; 118:2443-9.

4. Lang DM, Butz AM, Duggan AK, Serwint JR. Physical activity in urban school-aged children with asthma. Pediatrics 2004; 113:e341-6.

5. Lai KY, Lam Karry K L, Lam SC, et al. Exploring parents' understandings and concerns on self-management of childhood asthma. Hong Kong Practitioner 2005; 27:172-8.

6. Ministry of Health, Singapore. Management of Asthma. In: Clinical Practice Guidelines [online]. Available at: http://www.moh.gov.sg/content/moh_ web/home/Publications/guidelines/cpg/2008/management_of_asthma. html. Accessed June 24, 2013.

7. Lai CKW, Kim YY, Kuo SH, Spencer M, Williams AE, and on behalf of the Asthma Insights and Reality in Asia Pacific Steering Committee. Cost of Asthma in the Asia-Pacific region. Eur Respir Rev 2006; 15:10-6.

8. Hazir T, Das C, Piracha F, Waheed B, Azam M. Carers' perception of childhood asthma and its management in a selected Pakistani community. Arch Dis Child 2002; 87:287-90.

9. Wong E, Wong TW, Chung M, Lau CC. Knowledge and beliefs of parents of asthmatic children presenting to an emergency department. Hong Kong J Emerg Med 2001; 6:202-6.

10. Smeeton NC, Rona RJ, Gregory J, White P, Morgan M. Parental attitudes towards the management of asthma in ethnic minorities. Arch Dis Child 2007; 92:1082-7.

11. Shaw A, Thompson EA, Sharp D. Complementary therapy use by patients and parents of children with asthma and the implications for NHS care: a qualitative study. BMC Health Serv Res 2006; 6:76.

12. Sicherer SH, Sampson HA. Food allergy. J Allergy Clin Immunol 2006; 117 (2 Suppl Mini-Primer):S470-5.

13. Wicher IB, Ribeiro MA, Marmo DB, et al. Effects of swimming on spirometric parameters and bronchial hyperresponsiveness in children and adolescents with moderate persistant atopic asthma. J Pediatr (Rio J) 2010; 86:384-90.

14. Bernard A, Carbonnelle S, Michel O, et al. Lung hyperpermeability and asthma prevalence in school children: unexpected associations with the attendance at indoor chlorinated swimming pools. Occup Environ Med 2003; 60:385-94. 